\title{
Assessment of the Epidemiological Surveillance System for Bacterial Meningitis in Benin from 2016 to 2018: Case-by-Case Surveillance
}

\author{
Togbemabou Primous Martial Godjedo ${ }^{1,2}$, Moussiliou Noël Paraiso ${ }^{3}$, \\ Alidehou Jerrold Agbankpe ${ }^{4, *}$, Tamegnon Victorien Dougnon ${ }^{4}$, Yves Eric Denon ${ }^{2}$, Marie Hidjo ${ }^{4}$, \\ Honore Bankole ${ }^{2,4}$ \\ ${ }^{1}$ Epidemiological Surveillance Service, Ministry of Public Health, Cotonou, Benin \\ ${ }^{2}$ National Health Laboratory, Ministry of Public Health, Cotonou, Benin \\ ${ }^{3}$ Regional Institute of Public Health, University of Abomey-Calavi, Cotonou, Benin \\ ${ }^{4}$ Research Unit in Applied Microbiology and Pharmacology of Natural Substances, Research Laboratory in Applied Biology, Polytechnic \\ School of Abomey-Calavi, University of Abomey-Calavi, Cotonou, Benin
}

Email address:

agbankpejerrold@yahoo.fr (A. J. Agbankpe)

${ }^{*}$ Corresponding author

\section{To cite this article:}

Togbemabou Primous Martial Godjedo, Moussiliou Noël Paraiso, Alidehou Jerrold Agbankpe, Tamegnon Victorien Dougnon, Yves Eric Denon, Marie Hidjo, Honore Bankole. Assessment of the Epidemiological Surveillance System for Bacterial Meningitis in Benin from 2016 to 2018: Case-by-Case Surveillance. World Journal of Public Health. Vol. 5, No. 3, 2020, pp. 53-59. doi: 10.11648/j.wjph.20200503.12

Received: July 31, 2020; Accepted: August 17, 2020; Published: August 27, 2020

\begin{abstract}
With the introduction of the new meningitis A conjugate vaccine in 2012, Benin has opted for case-by-case surveillance for bacterial meningitis. The study aims to assess the case-by-case surveillance system for the meningitis epidemic in Benin during the period 2016 to 2018. A retrospective and evaluative study with a mixed approach (qualitative and quantitative) was conducted, on the three sites identified for sentinel surveillance of meningitis in Benign. The evaluation of the performance of the surveillance system was based on the updated guidelines of the Center for Disease Prevention and Control to evaluate a public health surveillance system. All criteria except sensitivity, specificity and positive predictive value were measured. Semi-structured individual interviews were conducted with the agents of the surveillance system surveyed and the normative documents as well as the notice forms were examined. This study reveals that all the centers had the case definition and notification forms. The ratio of notified cases to registered cases was 0.77 . We found that the monitoring system in place had five levels. The system performance was recognized with simplicity at $80.8 \%$, good acceptability (completeness: 98.5\%; Promptitude: $88.9 \%$ ) and responsiveness of $85 \%$. The completeness of the health facilities was $45 \%$. This study shows that it is imperative to strengthen the knowledge of the actors involved in epidemiological surveillance through periodic training to improve the performance of the surveillance system.
\end{abstract}

Keywords: Surveillance System Evaluation, Meningitis Epidemic, Benin from 2016 to 2018, Case-by-Case Surveillance

\section{Introduction}

Among the potentially epidemic diseases, meningococcal cerebrospinal meningitis remains a cyclical threat in Africa, particularly in the meningitis belt area. The dry season combined with the harmattan, the movements and habits of the populations favor its transmission. Vaccination with polysaccharide vaccines (bivalent AC, trivalent ACW135), accessible in the area does not allow obtaining sufficient "collective immunity" [1]. Indeed, these vaccines protect those who have received them, but do not lead to the development of antibodies in the nasopharynx and therefore do not stop the circulation of the bacteria. In addition, they cannot be used before the age of two, and after 30 years, individuals are off target for vaccination campaigns. The immunity granted lasts only three to five years [2]. These 
vaccines are not included in the expanded vaccination programs of meningeal belt countries and their use in mass campaigns is only in response to the declared epidemic [3]. In order to cope with the heavy morbidity and mortality linked to the disease, the WHO strategy, relayed by the ministries in charge of health in the affected countries, is to work on preparing for and responding to epidemics [4, 5].

For about a century, sub-Saharan Africa has experienced repeated epidemics of meningitis. Benin, a country located in West Africa, has made significant progress in the fight against vaccine-preventable diseases by significantly increasing its routine immunization coverage [6]. The northern zone of Benin is in the meningitis belt of Lapaysonnie, with a dry season from November to May during which the Harmattan blows, bringing dust and sand storms [7]. Since 1996 and almost every year, epidemics of meningococcal meningitis have been recorded, leading to deaths and various complications. Meningococcal epidemics disrupt the economy and the social organization of the affected localities. Because during an epidemic the local economy is at a standstill and social activities (pilgrimages, traditional local markets) are prohibited or restricted. The meningitis season in Benin extends from November to May [6].

The epidemiological point from 2003 to 2011 reveals 3,493 cases and 687 deaths, i.e. a lethality of $19.66 \%$. Most of the meningitis epidemics in Benin during these years were due to meningococcus serogroup A [6]. This led Benin to introduce the MenAfriVac vaccine in 2012. Since then, caseby-case surveillance has been put in place with the identification of three (03) sentinel sites installed in the cities of Natitingou, Parakou and Abomey.

This study aims to assess the performance of the case-bycase surveillance system for meningitis in the three sentinel surveillance sites in Benin, for a better response against the disease.

\section{Main Body}

\subsection{Conceptual Frame}

Figure 1 presents the conceptual framework adapted to the epidemiological surveillance system for meningitis in Benin (Case-by-case surveillance). First, the relationship between the structure of the system and its process is marked by the fact that:

1) normative resources define the essential functions of the process;

2) implementation of the elements of the process depends on the availability of human and material resources;

3 ) flow of data conditions the collection of data;

4) funding plays an important role in the choice of the surveillance operational strategy and plan;

5) system partners can contribute to data collection.

Then, the following relationships translate the link between the system process and its performance:

1) operationalization plan for surveillance conditions the completeness and representativeness of the system;

2 ) information produced and disseminated by the system makes it possible to judge its usefulness;

3) data collection procedure influences the simplicity of the system;

4) agent training plays an important role in the completeness and timeliness of data;

5) internal communication (Feedback) improves the acceptability of the system.

Finally, the structure of the system affects its performance because:

1) financing of the system conditions its stability;

2) human resources judge the acceptability and simplicity of the system;

3) availability of an internet or intranet connection, which is a material resource, can improve the responsiveness of the system.

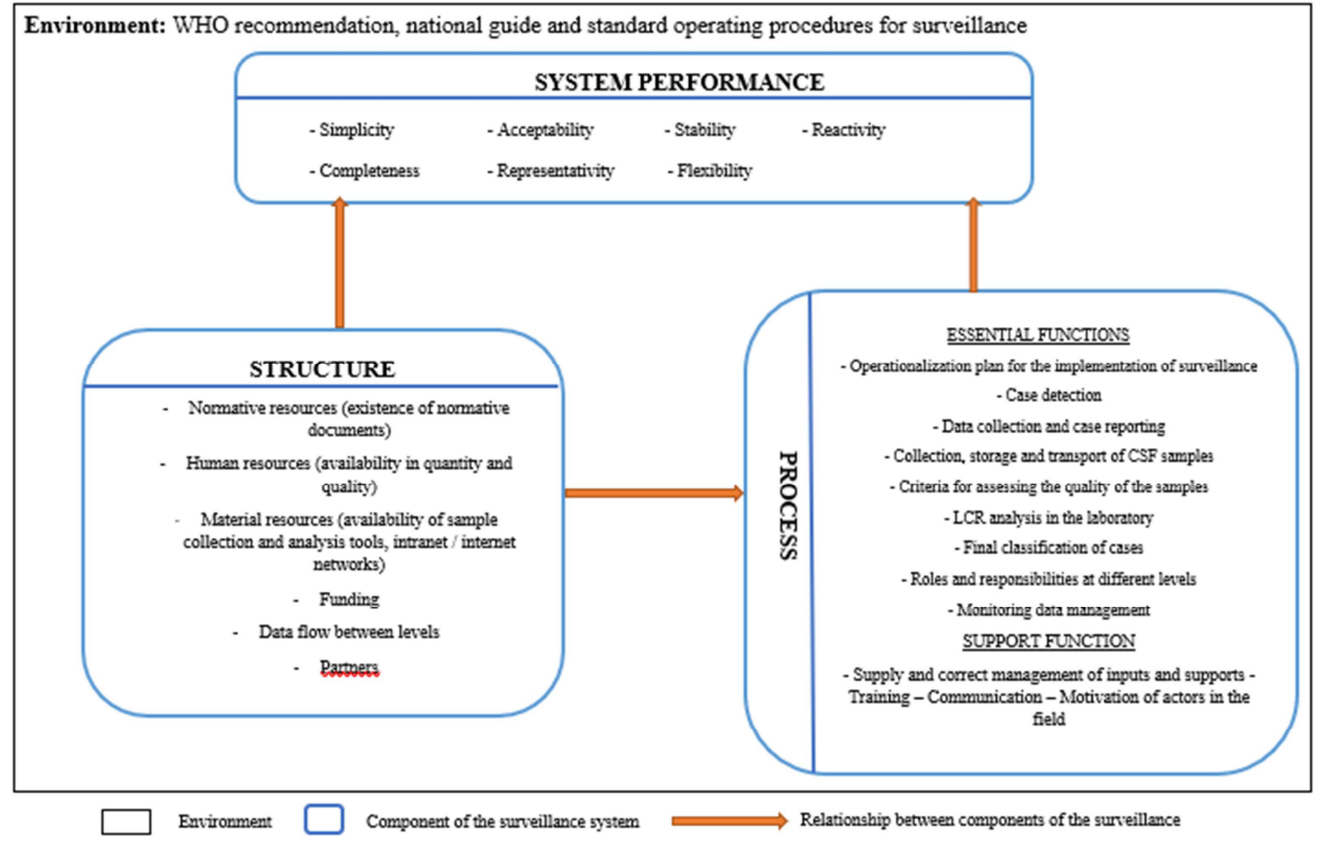

Figure 1. Conceptual framework of the epidemiological surveillance system for meningitis in Benin: case-by-case surveillance. 


\subsection{Materials and Methods}

This is a retrospective, evaluative study with a mixed approach (qualitative and quantitative) carried out on the epidemiological surveillance sites for meningitis (Figure 2) that are the University Hospital Centers of the Departments (CHUD) of "Borgou" (covers departments of "Borgou" and "Alibori") and "Atacora" (covers the departments of "Atacora" and "Donga") and the University Hospital Center (CHU) of "Goho" (covers the departments of "Zou" and "Collines"). Data collection took place from August 1 to December 30, 2019. It related to the examination of the files of all cases of meningitis registered (main target) between 2016 and 2018, i.e. 2992 cases, and on the actors of the surveillance system (secondary target), thus involving 81 health workers involved in the epidemiological surveillance of meningitis. On the one hand, these were the operational actors, which are the health agents responsible for taking samples and filling in the weekly and monthly epidemiological statements of the health units, the laboratory technicians in the health zones and, on the other hand, the strategic actors. of the surveillance system that are the area supervisors, the departmental directors of public health, the head of the National Reference Laboratory, the head of the epidemiology department of the National Health Department. The circuit of information and data relating to epidemiological surveillance was traced through these interviews.

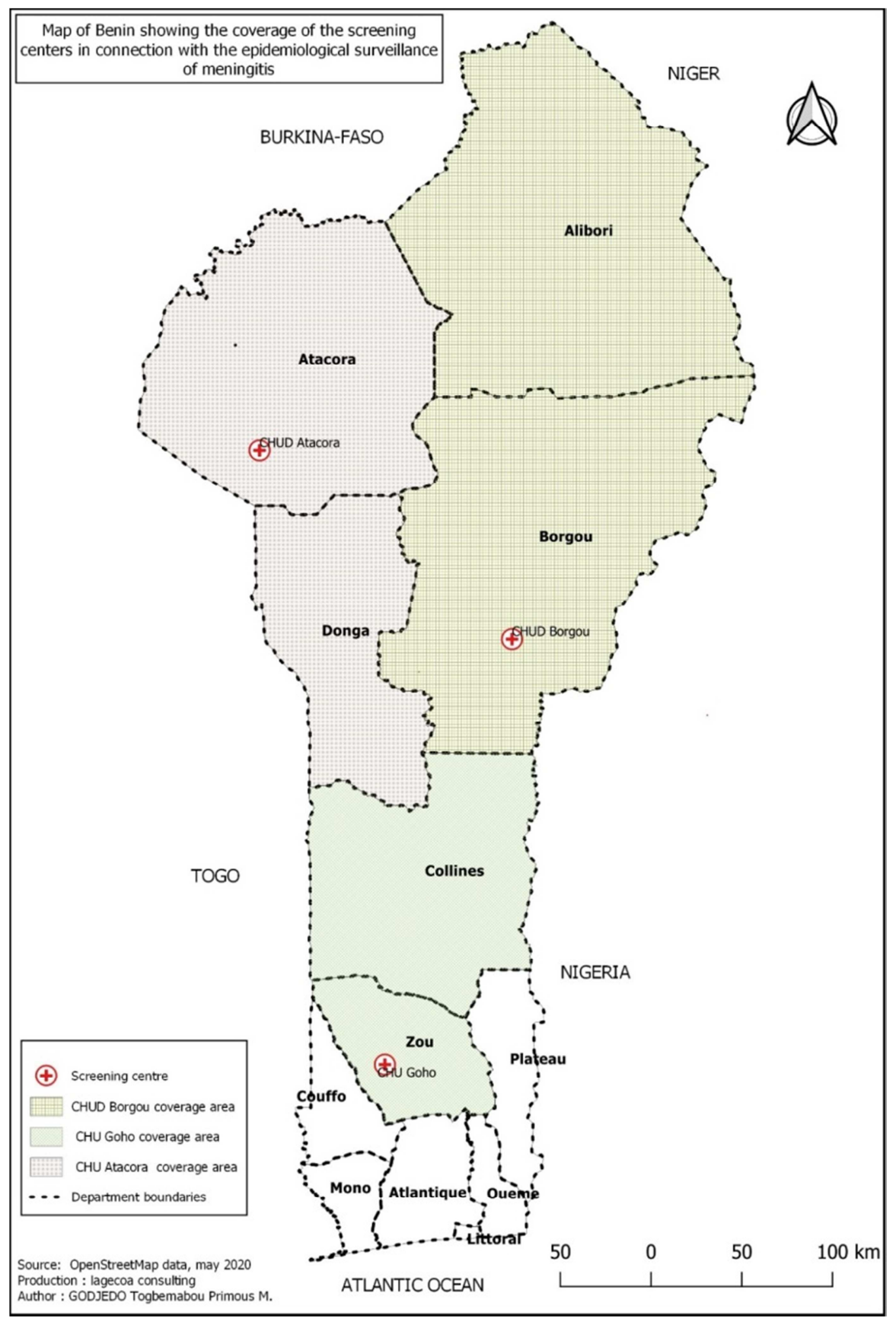

Figure 2. Map of Benin showing the three sentinel surveillance sites for the meningitis epidemic in Benin. 


\subsubsection{Sampling and Data Collection}

The sampling method for the strategic actors of the surveillance system was non-probabilistic with a reasoned choice sampling technique relating to the agents actually involved in surveillance and the heads of the services in charge of surveillance, because they are who hold the information sought. Thus, data collection was done by structured individual interviews based on an established questionnaire. As for the documentary review based on the examination of files for the period 2016 to 2018, there was talk of analyzing descriptive lists of cases, notification forms (duplicate), weekly reports, admission and laboratory registers. There was a digital Epi Info platform with the possibility of exporting data in Excel format to the three sentinel surveillance sites.

\subsubsection{Data Processing}

The data were entered into an Excel spreadsheet, processed and analyzed using statistical analysis software R.

\subsubsection{System Performance Analysis}

The case definition of cerebrospinal meningitis used in these CHUD and CHU is:

1) suspicious case: any adult patient with a sudden onset fever $\left(>38.5^{\circ} \mathrm{C}\right.$ rectal temperature or $38.0^{\circ} \mathrm{C}$ axillary temperature) with one of the following signs: stiff neck, neurological disorder or any other sign meningeal. Or any child with a sudden onset fever $\left(>38.5^{\circ} \mathrm{C}\right.$ rectal temperature or $38.0^{\circ} \mathrm{C}$ axillary temperature) with one of the following signs: stiff neck or soft neck, bulging fontanel, capping look, convulsion or any other meningeal sign;

2) probable case: any suspect case in which the lumbar puncture (PL) brings back a cerebrospinal fluid (CSF) of macroscopic, cloudy, purulent or xanthochromic appearance or the presence of Gram negative diplococci, Gram positive diplococci, Gram positive bacilli microscopic examination, or if the leukocyte count is greater than 10 cells $/ \mathrm{mm}^{3}$;

3) Confirmed case: any suspected or probable case in which the causative agent (Neisseria meningitidis, Streptococcus pneumoniae, Haemophilus influenzae b, etc.) were demonstrated by culture from the CSF or by Hemoculture or by PCR.

It is also emphasized that in the event of an epidemic, PL should not delay the start of treatment. The bacteriological diagnosis in the CHUD and CHU laboratories included direct examination and the possibility of culture. LCR agglutination and polymerase chain reaction (PCR) techniques were not available.

For the detection of the germ involved in the epidemic, and according to the National Guidelines, in the pre-epidemic phase, information on the cases detected was collected on individual notification forms from the Integrated Disease Surveillance and Response (SIMR) and sent with a CSF sample, via the CHUD and / or CHU laboratories to the National Reference Laboratory, responsible for determining the germ in question. For this period of epidemiological surveillance, in the epidemic phase, CSF samples were regularly sent to the National Laboratory from the departments for the monitoring of the germ.

The performance of the surveillance system was assessed according to the criteria: accessibility, which is the proportion of health facilities with case definition, case notification form, treatment protocol, shortage of notification form, proportion, health units whose staff have been trained; the effectiveness criteria that are: the ratio of the number of files notified to the number of cases recorded, reported lethality, age-specific lethality; the data quality criteria made up of the proportion of files providing information on age, the consultation period, the proportion of cases treated according to the protocol. In addition to these criteria, a few attributes were also used to assess the performance of the surveillance system [8, 9]. These include simplicity, acceptability, representativeness, responsiveness, stability and comprehensiveness.

\section{Results}

After monitoring, the surveillance system in place consisted of five levels: the level of health establishment made up of health centers located at the level of the commune, zone hospitals, departmental hospitals, university hospitals and private clinics (level 1) in charge of collecting meningitis data from consultation registers; the level of the health zone office (level 2) responsible for the compilation of data from health establishments by the Head of the Epidemiological Surveillance Center (ESC) before their transmission to the Departmental Health Directorate; the departmental level (level 3) where the data from the various health zone offices under its responsibility are validated, aggregated and transmitted to the National Directorate of Public Health located at the national level, the national level (level 4) compiles the data from the departments. Then these data are verified, analyzed and interpreted before their transmission to the country office of the World Health Organization (WHO) which constitutes level 5 through an epidemiological bulletin. The interpretation of this data at different levels is the basis for decision-making and the implementation of public health measures. The reactivity (Table 1) was 85\% (95\% CI: 0.79 - 0.82). The score concerning the time between sampling and the availability of results was $76.8 \%$ (Table 1 ).

Table 1. Evaluation of the reactivity of the case-by-case surveillance system for meningitis in Benin from 2016 to 2018.

\begin{tabular}{ll}
\hline Reactivity $\%=85$ and $\mathbf{9 5} \% \mathbf{C I}[\mathbf{0 , 7 9}-\mathbf{0 , 8 2}]$ & $\mathbf{\%}$ \\
\hline Existence of rapid means of communication & 100 \\
Time between samples and availability of results & 76.8 \\
Time between notification and investigation of cases & 100 \\
\hline
\end{tabular}

All health workers interviewed in health facilities stated that they always have the standard definition of the case of meningitis and the case notification sheets, before and during 
the epidemic. Acceptability during the study period (2016 to 2018) allowed to observe an average completeness of $98.5 \%$ and an average promptness of $88.9 \%$ (Table 2 ).

Table 2. Percentage of acceptability indicators based on each year of the considered epidemiological surveillance period (2016 to 2018).

\begin{tabular}{lllll}
\hline Acceptability & \multicolumn{3}{l}{ Epidemiological surveillance period } & \multirow{2}{*}{ Moyenne } \\
\cline { 2 - 4 } indicators (\%) & $\mathbf{2 0 1 6}$ & $\mathbf{2 0 1 7}$ & $\mathbf{2 0 1 8}$ & \\
\hline Completeness & 100 & 96.7 & 98.7 & 98.5 \\
Promptness & 88 & 91.8 & 86.9 & 88.9 \\
\hline
\end{tabular}

Out of a total of 2,857 registered cases, $98.2 \%$ of the notification form were found in the structures around one year later. The ratios of the number of cases notified at the departmental level and at the national level, over the number of cases registered were 0.99 and 0.77 respectively (Table 3 ). $95.6 \%$ of the files indicated the age of the patients, but only $90.6 \%$ of the files specified the time taken to consult cases. The patients' place of residence was mentioned in $86.9 \%$ of the files. The delivery time for the files at the departmental level was regular every seven days.

Table 3. Overview of registration and notification of cases during epidemiological surveillance of meningitis from 2016 to 2018 in Benin.

\begin{tabular}{|c|c|c|c|c|}
\hline & \multicolumn{3}{|c|}{ Epidemiological surveillance sites for meningitis } & \multirow{2}{*}{ Total } \\
\hline & CHUD Borgou & CHUD Alibori & CHU Goho & \\
\hline Recorded case (E) & 678 & 2165 & 14 & 2857 \\
\hline Notification form found & $660(97.3 \%)$ & $2132(98.5 \%)$ & $14(100 \%)$ & $2806(98.2 \%)$ \\
\hline Cases notified at regional level (N1) & 672 & 2159 & 12 & 2843 \\
\hline Case notified to DNSP (N2) & 433 & 1746 & 10 & 2189 \\
\hline Ratio 1 (N1/E) & 0.99 & 0.99 & 0.86 & 0.99 \\
\hline Ratio 2 (N2/E) & 0.64 & 0.81 & 0.71 & 0.77 \\
\hline
\end{tabular}

CHUD: Departmental University Hospital Center; CHU: University Hospital Center; DNSP: National Directorate of Public Health.

An average level of simplicity of $80.8 \%$ (95\% CI: $0.65-$ $0.98)$ was observed with an average knowledge of health personnel on the alert thresholds, i.e. $52.8 \%$ and a low level of $42.5 \%$ compared to the epidemic threshold (Table 4 ).

Table 4. Assessment of the simplicity of implementing the case-by-case surveillance system for meningitis in Benin from 2016 to 2018.

\begin{tabular}{ll}
\hline Simplicity $(\mathbf{\%})=\mathbf{8 0 , 8}(\mathbf{9 5} \% \mathbf{C I}: \mathbf{0 , 7 5}-\mathbf{0 , 8 2})$ & $\mathbf{\%}$ \\
\hline Availability of monitoring agents & 100 \\
Use of case definition for notification of suspected meningitis cases & 100 \\
Knowledge of the alert threshold & 52.8 \\
Knowledge of the epidemic threshold & 42.5 \\
Availability of the epidemiological surveillance guide & 62.7 \\
Availability of meningitis guidelines & 95.8 \\
Availability of notification form & 100 \\
Standardized data transmission & 100 \\
\hline
\end{tabular}

Regarding to the exhaustiveness of the case-by-case surveillance system, the database of the National Public Health Department mentioned 104 confirmed cases of meningitis during the surveillance period considered (20162018) while that of all three surveillance sites investigated evoked 899 cases (Table 5). The total number of cases estimated by the capture method was 1429 (95\% CI: 13961462). The completeness of all monitoring sites was $62.9 \%$ and that of the DNSP was $7.3 \%$ (Table 5).

Table 5. Completeness of the case-by-case surveillance system for meningitis in Benin from 2016 to 2018.

\begin{tabular}{|c|c|c|c|c|}
\hline & & \multicolumn{3}{|c|}{ Number of meningitis cases reported by surveillance sites mandatory reporting } \\
\hline & & + & - & Total \\
\hline \multirow{3}{*}{$\begin{array}{l}\text { Number of meningitis cases } \\
\text { reported by the DNSP }\end{array}$} & + & 97 & 7 & 104 \\
\hline & - & 802 & 523 & 1325 \\
\hline & Total & 899 & 530 & 1429 \\
\hline
\end{tabular}

$+=$ confirmed case of meningitis; -=unconfirmed case of meningitis; DNSP=National Directorate of Public Health.

\section{Discussion}

The limits of our study are inherent to those of retrospective studies (incomplete documents, archiving problems...). Nevertheless, it has raised interesting questions in the management of meningitis epidemics in the various municipalities. It can thus be noted that the case-by-case epidemiological surveillance system set up in health establishments is accessible and well functional. Monitoring tools are available at all levels and case definition is within the reach of officers; no shortage of notice was noted. However, the ratio of the number of cases notified to the number of cases registered is less than $1(0.77)$, reflecting under-reporting of cases. Similarly, the registration of cases and the updating of the database are insufficient. The poor quality of surveillance in health facilities in Africa is conventionally reported in the literature [10-12]. However, the inadequacies of archiving (premises, furniture, bad weather, etc.), the insufficiency of human resources usually observed in health establishments in the northern zone of 
Benin, with the multiplication of the workload due to management other epidemics other than meningitis, could contribute to the loss of information and to the filling in of certain items on the meningitis case notification sheets. It is important to know key items such as the age of the patient, their place of residence and their time to consult after the onset of symptoms. This makes it possible to describe the profile of cases, to list the areas affected by the epidemic and to assess the impact of awareness-raising messages, regarding the early attendance of centers $[1,12]$.

Compared to the functioning of the surveillance system, its average simplicity was $80.8 \%$, with little knowledge of the epidemic threshold (42.5\%) and an average knowledge of the alert threshold $(52.8 \%)$ (Table 4). These results are comparable to those obtained by Djila et al., where the simplicity of the epidemiological surveillance system for meningitis in the health district of commune VI of Bamako from 2011 to 2013 was $81.86 \%$ with little knowledge of the alert threshold (47.1\%) and an average knowledge of the epidemic threshold (51\%) [13]. We found that the availability of meningitis guidelines was $95.8 \%$ and that of the national technical guide to integrated disease surveillance and response $62.7 \%$. These results show that the structure of the case-by-case epidemiological surveillance system for meningitis in Benin is light, but must be reinforced by training agents on knowledge of the alert and epidemic threshold as well as updating the national guide. and standard operating procedures for case-by-case surveillance of bacterial meningitis in Benin and its availability at the level of all the hospitals involved in epidemiological surveillance. Our results are comparable to those found by Dolo in the 2011 Katide evaluation with the availability of meningitis guidelines which was $96.4 \%$ [14].

How responsive was the surveillance system? The average reactivity observed in this study was $85 \%$. The deadline for compiling the files, analyzing and transmitting the data was one week, from the health establishments to the epidemiological surveillance center of the health zone office. Can we be satisfied with a weekly notification in the surveillance of meningitis at the level of health establishments? The strengthening of the surveillance system should tend to reduce this period to health establishments for better preparation for epidemics and response. The means of communication, such as the mobile phone, the social network whatsApp, generally available in health facilities, should currently be used to compile daily data from these health establishments, to a center, the office's epidemiological surveillance center. of the sanitary area. In the short term, the surveillance system for diseases with epidemic potential would benefit from the establishment of computerized registers in health facilities [1].

The acceptability of the functioning of epidemiological surveillance was shown through an average completeness of $98.5 \%$ and an average of promptnesses of $88.9 \%$. This shows that the actors are strongly involved in the surveillance system with a high participation rate and a lot of time devoted to collecting and transferring information.
Motorcycles are used to transport CSF samples from peripheral health centers to surveillance laboratories. Although precautions are usually taken to keep these samples in good temperature conditions, their quality on arrival is dependent on the delivery time. Transport is an important element, the meningococcus being a very fragile germ. This could explain the large number of samples found to be defective in the laboratory. In addition, the insufficiency of human resources, that of equipment (weakness of storage capacity for samples) and laboratory inputs (culture media) could also account for part of the wide gap observed between the number of CSF samples received and the number actually examined. The interviews revealed that in several health establishments in the northern zone, doctors to rule out meningitis in suspected patients systematically make a lumbar portion. This state of affairs pushes the epidemiological surveillance agents to mention these cases as suspect cases, which means that some municipalities are constantly in an epidemic situation.

\section{Conclusion}

This study shows an acceptable functioning of the case by case surveillance of meningitis in Benin. Despite the existence of a functional surveillance and supervision system, there is an under-notification of cases, a poor knowledge of alert and epidemic thresholds and a weak contribution of the laboratory in the identification of germs. It is therefore imperative to strengthen the knowledge of actors involved in epidemiological surveillance through periodic training. In addition, the capacity of diagnostic laboratories must be strengthened by providing them with materials and equipment for rapid and efficient diagnostics. Finally, an update of the national guide and standard operating procedures for case-bycase surveillance of bacterial meningitis in Benin published in September 2013 and its availability at the level of all the health establishments involved in epidemiological surveillance would be essential for improving case-by-case surveillance of meningitis in Benin.

\section{Acknowledgements}

The authors thank all the staff of the epidemiological surveillance service of the Ministry of Health of Benin and all the health personnel who participated in this study.

\section{References}

[1] T. M. Yaméogo, C. G. Kyelem, G. E. A. Poda, I. Sombié, M. S. Ouédraogo, A. Millogo (2010). Meningitis epidemic: assessment of surveillance and treatment of cases in the health centers of a Burkina Faso district. Bull. Soc. Pathol. Exot., 1-6, DOI 10.1007/s13149-010-0123-3.

[2] C. Lingani, C. Bergeron-Caron, J. M. Stuart, et al (2015). Meningococcal meningitis surveillance in the African meningitis belt, 2004-2013. Clin Infect Dis; 61 (Suppl 5): S410-5. 
[3] C. L. Trotter, C. Lingani, K. Fernandez, et al (2017). Impact of MenAfriVac in nine countries of the African meningitis belt, 2010-15: an analysis of surveillance data. Lancet Infect Dis; 17: 867-72.

[4] World Health Organization and Centers for Disease Control and Prevention. Technical guidelines for integrated disease surveillance and response in the African region. Available at: https://www.cdc.gov/globalhealth/healthprotection/idsr/pdf/te chnicalguidelines/idsr-technical-guidelines-

2ndedition_2010_english.pdf. Accessed 22 October 2019.

[5] World Health Organization (2019). Epidemic meningitis control in countries in the African meningitis belt, 2018. Wkly Epidemiol Rec; 94: 179-88.

[6] National Directorate of Public Health. National guide and standard operating procedures for case-by-case surveillance of bacterial meningitis. Ministry of Public Health of Benin-WHO September, 2013, $80 \mathrm{p}$.

[7] T. P. Godjedo, M. N. Paraiso, A. J. Agbankpe, T. V. Dougnon, C. Degbey, A. Ahoyo, et al (2020). Case-ByCase Surveillance for Bacterial Meningitis in Benin: Data Analysis, 2016 to 2018. Open Journal of Epidemiology; (10): $1-14$.

[8] Madjid Herida. Methods for evaluating public health surveillance systems. Public health and epidemiology. Université Paris-Saclay, 2016. Français. NNT: 2016SACLS279.
[9] Centers for Disease Control and Prevention. Framework for evaluating public health surveillance systems for early detection of outbreaks: recommendations from the CDC working group. Mortality Morbidity Weekly Report [online]. 2004 [consulté le 15/10/2019]; (53 ( $\mathrm{N}^{\circ}$ RR-5)): 1-13. Disponible:

http://www.cdc.gov/mmwr/preview/mmwrhtml/rr5305a1.htm.

[10] H. Veeken, K. Ritmeijer, B. Hausman (1998). Priority during a meningitis epidemic: vaccination or treatment? Bull World Health Organ, 76 (2): 135-41.

[11] G. Mengistu, K. Mitiku, W. Teferi (2003). Analysis and reporting of meningococcal meningitis epidemic in north Gondar 2001- 2002. Ethiop Med J, 41 (4): 319-31.

[12] S. Taylor, L. Shimp (2010). Using data to guide action in polio health communications: experience from the Polio Eradication Initiative (PEI). Health Commun, 15 Suppl 1: 48-65.

[13] M. Djilla, M. Konake, K. Kayentao, M. Sacko, A. Ag Iknane, D. Sissoko (2014). Evaluation of the epidemiological surveillance system for meningitis in the health district of commune VI of Bamako from 2011 to 2013. Mali Public Health, Tome 4, Nº 001 \& 002: 25-27.

[14] A. Dolo (2012). Evaluation of the epidemiological surveillance system for meningitis in the Kati health district in 2011. Bamako (Mali): University of Bamako. 